\title{
Richness and density of aquatic benthic macroinvertebrates after exposure to fungicides and insecticides in rice paddy fields
}

\author{
ALANA C.D. WANDSCHEER ${ }^{1}$, ENIO MARCHESAN ${ }^{1}$, SANDRO SANTOS ${ }^{2}$, RENATO ZANELLA \\ MARÍLIA F. SILVA ${ }^{1}$, GUILHERME P. LONDERO ${ }^{4}$ and GABRIEL DONATO ${ }^{1}$ \\ ${ }^{1}$ Universidade Federal de Santa Maria, Centro de Ciências Rurais, Departamento de Fitotecnia, Avenida \\ Roraima, 1000, Prédio 77, Cidade Universitária, Camobi, 97105-900 Santa Maria, RS, Brazil \\ ${ }^{2}$ Universidade Federal de Santa Maria, Centro de Ciências Naturais e Exatas, Departamento de Ecologia e Evolução, \\ Avenida Roraima, 1000, Prédio 17, Cidade Universitária, Camobi, 97105-900 Santa Maria, RS, Brazil \\ ${ }^{3}$ Universidade Federal de Santa Maria, Departamento de Química, Laboratório de Análises de Resíduos de Pesticidas/ \\ LARP, Avenida Roraima, 1000, Prédio 18, Cidade Universitária, Camobi, 97105-900 Santa Maria, RS, Brazil \\ ${ }^{4}$ Universidade Federal de Santa Maria, Centro de Ciências Rurais, Departamento de Defesa Fitossanitária, \\ Avenida Roraima, 1000, Prédio 42, Cidade Universitária, Camobi, 97105-900 Santa Maria, RS, Brazil
}

Manuscript received on August 29, 2016; accepted for publication on October 7, 2016

\begin{abstract}
The objective of this study was to verify the richness and density of aquatic benthic macroinvertebrates after exposure to fungicides and insecticides of the rice paddy fields. In the crop seasons of 2012/13 and 2013/14, field experiments were performed, which consisted of single-dose applications of the fungicides trifloxystrobin + tebuconazole and tricyclazole, and the insecticides lambda-cyhalothrin + thiamethoxam and diflubenzuron, in $10 \mathrm{~m}^{2}$ experimental plots, over rice plants in the R3 stage. Control plots with and without rice plants were maintained in order to simulate a natural environment. Soil samples were collected during rice cultivation for assessment of the macroinvertebrate fauna. Chemical-physical parameters assessed in the experiments included temperature, $\mathrm{pH}$ and oxygen dissolved in the water and pesticide persistence in the water and in the soil. The application of a single dose of the pesticides and fungicides in the recommended period does not cause significant negative effects over the richness and density of the macroinvertebrates. Tebuconazole, tricyclazole and thiamethoxam showed high persistence in the irrigation water of rice paddy fields. Thus, the doses and number of applications of these products in crops should be carefully handled in order to avoid contamination of the environment.
\end{abstract}

Key words: aquatic invertebrates, lowlands, Oryza sativa L., pesticides.

\section{INTRODUCTION}

Rice fields are environments with high biologic diversity compared to other agricultural areas (Stenert et al. 2012) and conservation of species in these areas may be an important alternative for

Correspondence to: Alana Cristina Dorneles Wandscheer

E-mail: alanacdw@hotmail.com biodiversity conservation in wetlands (Maltchik et al. 2011). However, the intense use of pesticides in rice crop management may cause major impacts over non-target organisms, as rice fields are considered temporary aquatic environments which favor the development of many species (Bambaradeniya et al. 2004). 
The use of pesticides modifies food webs and the development of communities (Mesléard et al. 2005). Traditionally, the assessment of environmental aspects in aquatic ecosystems is performed by means of measurements of changes in the concentration of physical and chemical variables. However, this technique is little efficient in detecting diversity changes in habitats and microhabitats and insufficient for determining consequences of changes in the water quality in biological communities, which suggests biologic monitoring as the most reliable tool in the assessment of responses of the biologic communities to changes in the environment (Goulart and Callisto 2003).

Characterization of a community is a good indicator for environmental changes, as a result of exposure to pesticides (Liess et al. 2008). In this sense, the assessment of a bioindicator community is recommended for assessing the impact in an agroecosystem (Gamboa et al. 2008). Benthic macroinvertebrates are among the most indicated organisms, because they present population abundance and easy identification, broad distribution in freshwater ecosystems and sensibility to perturbation factors. In addition, these organisms live in the sediments that tend to accumulate pesticide residues, which potentiate the effects of such products (Gamboa et al. 2008).

Many fungicides and insecticides are currently used for pest and disease control in rice paddy fields, and, the most common fungicides and pesticides belong to the groups of strobilurins, triazoles, benzothiazoles, pyrethroids, neonicotinoids, and benzoylureas. In this context, the objective of this study was to verify the richness and density of aquatic benthic macroinvertebrates community after exposure to fungicides and insecticides (trifloxystrobin, tebuconazole, tricyclazole, lambdacyhalothrin, thiamethoxam, and diflubenzuron) in rice paddy fields. These pesticides were chosen because of their broad use in rice paddy fields in the south of Brazil.

\section{MATERIALS AND METHODS}

The study was performed in an experimental area of wetlands of the Universidade Federal de Santa Maria (UFSM) (latitude -29.7000, longitude -53.7000 and altitude - 95 m), in Santa Maria, in Rio Grande do Sul state, in the south of Brazil, one of the biggest rice production areas in the world, for two crop seasons, 2012/13 and 2013/14, with a rice paddy field.

In each crop season, a field experiment was setup, consisting of six experimental plots with a total area of $10 \mathrm{~m}^{2}$ each $(4 \times 2.5 \mathrm{~m})$, whereas five plots had rice plants and one plot did not have rice plants, simulating the natural environment. Each experimental plot consisted of a treatment: $\mathrm{T} 1$ : application ofthecommercial formulationcontaining the active principles of fungicides trifloxystrobin + tebuconazole $\left(50+100 \mathrm{~g}\right.$ a.i. ha $^{-1}$, respectively); T2: application of the commercial product containing the active principle of the fungicide tricyclazole (225 g a.i. ha $\left.{ }^{-1}\right)$; T3: application of the commercial formulation containing the lambda-cyhalothrin + thiamethoxam insecticides $\left(15.9+21.15 \mathrm{~g}\right.$ a.i. $\mathrm{ha}^{-1}$, respectively); T4: application of the commercial product containing the active principle of the physiological insecticide diflubenzuron (19.2 g a.i. $\left.\mathrm{ha}^{-1}\right)$; T5: control with rice plants; T6: control without rice plants. The pesticides were applied only once in the plots over the shoot of the plants during the $\mathrm{R} 3$ reproductive stage (panicle exertion) of rice, according to the scale proposed by Counce et al. 2000, and also over the water layer in the plot without rice plants. The applications were performed with a $\mathrm{CO}_{2}$ backpack sprayer (40 lbs pol $^{-2}$ pressure) with $150 \mathrm{~L} \mathrm{ha}^{-1}$ spray volume, and a bar with four hollow cone nozzle tips (Jacto JA-2), spaced at $0.50 \mathrm{~m}$ and operating at $276 \mathrm{kPa}$.

The experimental plots were isolated by bunds and were irrigated and drained individually in order to avoid contamination with the different pesticides to be assessed. Sampling of macroinvertebrates 
inside each experimental plot was performed as repetitions, which were defined by the sampling sufficiency analysis, performed prior to the first collection (Wandscheer et al. 2016), whereas the minimum value found was 10 samples per plot.

For rice cultivation, the PUITÁ INTA CL cultivar was seeded at the density of $85 \mathrm{~kg} \mathrm{ha}^{-1}$ seed, in the minimum cultivation system. Basal fertilization, topdressing nitrogen, start of irrigation of the plots and other cropping features were performed in accordance with the soil analysis and technical recommendations for crops in rice paddy fields (SOSBAI 2012). When the experimental plots were irrigated, they remained with a water depth of $0.10 \mathrm{~m}$. In addition, a large, round bund was built in order to avoid water loss by lateral infiltration, maintaining the hydraulic load of the plots.

Soil collections were performed with the support of a cylindrical PVC Corer, with 0.1 $\mathrm{m}$ diameter, at a depth of $0.1 \mathrm{~m}$ from the soil, at four moments during the first year ( 1 day before pesticide application (day 0), 1 day after pesticide application (day 1), 15 and 30 days after pesticide application (days 15 and 30, respectively)). For the second year, an additional collection was performed within 7 days after pesticide application (day 7). These sample days were chosen in order to include the entire period of rice cultivation.

In the field, the soil samples were stored in labeled plastic bags, and then taken to the laboratory to be washed in running water, by means of a 0.5 mm mesh sieve (Kuhlmann et al. 2012). After washing, the samples were placed in $1 \mathrm{~L}$ plastic containers, in which $70^{\circ} \mathrm{GL}$ ethyl alcohol and Rose Bengal stain ( $1 \mathrm{~L}$ of $70^{\circ} \mathrm{GL}$ ethyl alcohol: $1 \mathrm{~g}$ of stain) were added, so that the final ratio was $1: 2$ (soil sample: stain $+70^{\circ} \mathrm{GL}$ ethyl alcohol).

The analyses of the samples were performed in two steps: trial and identification; count of existing taxa. In the trial step, the samples were washed once again in running water to clean off excess stain. Then, the samples were placed in white-bottom trays, and with the aid of a lamp, the thicker materials and bigger specimens were removed with the naked eye. Later, an analysis with a stereomicroscope was performed for removal of smaller individuals from the samples. The collected individuals were identified under stereomicroscopy up to the smallest possible taxonomic level, based on references in the literature (Fernández and Domínguez 2001, Costa et al. 2006, Mugnai et al. 2010).

The assessed parameters were richness (number of taxa) and density (number of specimens per square meter). Physical-chemical parameters of the water were assessed during the sampling days, such as temperature, $\mathrm{pH}$, dissolved oxygen and pesticide persistence. Pesticide concentration in the water was assessed $5 \mathrm{~min}$ after the first application (day 0 ) and 1 day later (day 1), 7 days later (day 7 - for the second year), 15 and 30 days after pesticide applications (days 15 and 30). Pesticide concentration in the soil was assessed 1 day later (day 1), 7 days later (day 7 - for the second year), 15 and 30 days after pesticide applications (days 15 and 30).

For water collection within 5 min after pesticide application (day 0), glass containers with $250 \mathrm{~mL}$ of ultra-pure water were used. They were put inside the plots immediately prior to the applications and removed $5 \mathrm{~min}$ later. For the other water samples (days 1, 7, 15 and 30), glass containers were used; they were cleaned with cleaning solution (Extran $\left.{ }^{\circledR}\right)$ and with the water to be sampled. The soil samples were collected with the support of core soil at a depth of $0.1 \mathrm{~m}$. The water and soil collected during the conduction of the experiments were assessed by the Pesticide Residue Analysis Laboratory (Laboratório de Análise de Resíduos de Pesticidas, LARP) at UFSM.

For the determination of pesticide persistence in the water, the samples were filtered in nylon membranes of $0.45 \mu \mathrm{m}$. Later, they were submitted to the pre-concentration process in Solid Phase 
Extraction (SPE) cartridges, containing $500 \mathrm{mg}$ of $\mathrm{C}_{18}$ followed by the elution with solvent (Donato et al. 2015). Pesticide analysis was performed by high-performance liquid chromatography with diode array detection (HPLC-DAD) (Caldas et al. 2011, Gonçalves et al. 2013).

The soil samples were extracted by employing the modified QuEChERS method, where $5 \mathrm{~g}$ of soil was extracted using $10 \mathrm{~mL}$ of acetonitrile acidified with $1 \%(\mathrm{v} / \mathrm{v})$ acetic acid followed by $1-\mathrm{min}$ agitation. Partition was performed using $3 \mathrm{~g}$ of anhydrous magnesium sulfate and sodium acetate followed by agitation and subsequent centrifugation. The extract was cleaned with anhydrous magnesium sulfate and PSA, with agitation and centrifugation. Pesticide determination was performed with ultrahigh-performance liquid chromatography and serial mass spectrometry (UHPLC-MS/MS) with electrospray ionization (ESI) sources (Prestes et al. 2009, Martins et al. 2014).

The data collected from the water physicalchemical parameters and density of the organisms was assessed for normality with the Shapiro-Wilk test, and with the Bartlett test for equal variances. For non-normal data, the data was transformed $(\sqrt{\mathrm{x}})$ for adequacy to the two-way analysis of variance (ANOVA two-way), which compared the variables (density and physical-chemical parameters of water) in the treatments and during the sampling days $\left(1^{\text {st }}\right.$ factor $=$ treatments and $2^{\text {nd }}$ factor $=$ sampling days). To complement the analysis, the Tukey's test was used at 5\% level of error probability. The data on density, water physical-chemical parameters and pesticide persistence in the water were correlated by means of linear correlation analysis. The statistical software used for making the analysis was BioEstat (5.0) (Ayres et al. 2007).

\section{RESULTS}

\section{WATER PHYSICAL-CHEMICAL PARAMETERS}

The means of dissolved oxygen in water showed little variation among the sampling days in both crop seasons. For the first year, the means ranged from 8.9 to $6.4 \mathrm{mg} \mathrm{L}^{-1}$ (Table I), and the highest values were found on the $15^{\text {th }}$ day after pesticide application. For the second year, the means ranged from 7.9 to $5.6 \mathrm{mg} \mathrm{L}^{-1}$, whereas the highest values were found on the $7^{\text {th }}$ day after pesticide application. There were no effects of the treatments on the dissolved oxygen in water in the experimental plots in either year of the study.

Water temperature ranged from 26.2 to $35.6{ }^{\circ} \mathrm{C}$ in the first year, and it was significantly higher on the days of pesticide application. In the second year, it ranged from 26.6 to $38.5{ }^{\circ} \mathrm{C}$, whereas the highest temperature was found on the $30^{\text {th }}$ day after pesticide application, compared to the other sampling days. Water temperature was also significantly higher in the control treatment without the presence of rice plants (T6) because in this environment, the absence of the rice plant canopy allowed higher light incidence, favoring the increase in water temperature.

Water $\mathrm{pH}$ ranged from 6.2 to 8.7 in the first year and from 6.1 to 6.9 in the second year, and there was no statistic difference among the treatments and sampling days for both study years.

There was a significant negative correlation between dissolved oxygen and water temperature, which was verified in T3 and T6 in the first year of study, and in T2, T3 and T4, in the second year. The $\mathrm{pH}$ value was also positively correlated with temperature in $\mathrm{T} 1, \mathrm{~T} 3$ and $\mathrm{T} 5$, in the second study year.

\section{PESTICIDE PERSISTENCE IN WATER AND SOIL}

Trifloxystrobin was detected in the water until 1 day after pesticide applications, but it was not detected in the soil, for both crop seasons (Table II). On the other hand, tebuconazole was found in the water until 30 days after the applications, in both years, and at a low concentration $(0.01 \mathrm{mg}$ $\left.\mathrm{kg}^{-1}\right)$ in the soil in the second year, 1 day after the applications. There was a positive correlation between water temperature and the concentrations 
TABLE I

Physical-chemical parameters in the water. DO: dissolved oxygen; $\mathrm{pH}$ : potential hydrogen; $\mathrm{T}$ : temperature; T1 - trifloxystrobin + tebuconazole; T2 - tricyclazole; T3 - lambda-cyhalothrin + thiamethoxam; T4 - diflubenzuron; T5 - control with rice plants; T6 - control without rice plants; day 0: 1 day before pesticide applications; day 1: 1 day after pesticide applications; day 7: 7 days after pesticide applications; day 15: 15 days after pesticide applications; day 30: 30 days after pesticide applications.

\begin{tabular}{|c|c|c|c|c|c|c|}
\hline \multicolumn{7}{|c|}{$\mathrm{DO}\left(\mathrm{mg} \mathrm{L}^{-1}\right)$} \\
\hline \multicolumn{7}{|c|}{ Crop season 2012/13 } \\
\hline Day & $\mathrm{T} 1$ (ns) & T2 (ns) & T3 (ns) & T4 (ns) & T5 (ns) & T6 (ns) \\
\hline $0(a b)^{*}$ & 7.2 & 7.4 & 6.9 & 7.1 & 7.2 & 6.9 \\
\hline $1(b)$ & 6.9 & 6.8 & 6.4 & 6.7 & 7.2 & 7 \\
\hline 15 (a) & 7.1 & 6.6 & 8.9 & 8.6 & 8.2 & 7.5 \\
\hline $30(a b)$ & 7.4 & 8.1 & 7.4 & 7.4 & 7.5 & 7.1 \\
\hline \multicolumn{7}{|c|}{ Crop season $2013 / 14$} \\
\hline Day & T1 (ns) & T2 (ns) & T3 (ns) & T4 (ns) & T5 (ns) & T6 (ns) \\
\hline $0(a b)$ & 7.3 & 7.2 & 7.6 & 7.3 & 7.6 & 7.7 \\
\hline $1(b)$ & 6.4 & 6.7 & 6.7 & 6.5 & 7.1 & 6.7 \\
\hline 7 (a) & 7.7 & 7.7 & 7.9 & 7.7 & 7.7 & 7.4 \\
\hline $15(b)$ & 6.9 & 6.3 & 6.7 & 6.6 & 7.3 & 6.2 \\
\hline $30(\mathrm{c})$ & 6.2 & 5.6 & 6.3 & 6.2 & 6 & 6.4 \\
\hline \multicolumn{7}{|c|}{$\mathrm{pH}$} \\
\hline \multicolumn{7}{|c|}{ Crop season $2012 / 13$} \\
\hline Day & T1 (ns) & T2 (ns) & T3 (ns) & T4 (ns) & T5 (ns) & T6 (ns) \\
\hline 0 (ns) & 7.1 & 6.9 & 6.8 & 6.9 & 6.9 & 7.2 \\
\hline $1(\mathrm{~ns})$ & 6.3 & 6.8 & 6.8 & 6.6 & 6.4 & 8.7 \\
\hline 15 (ns) & 6.7 & 6.2 & 6.4 & 6.5 & 6.6 & 6.8 \\
\hline $30(\mathrm{~ns})$ & 6.3 & 6.8 & 6.4 & 6.6 & 6.5 & 6.7 \\
\hline \multicolumn{7}{|c|}{ Crop season $2013 / 14$} \\
\hline Day & T1 (ns) & T2 (ns) & T3 (ns) & $\mathrm{T} 4$ (ns) & T5 (ns) & T6 (ns) \\
\hline 0 (ns) & 6.4 & 6.7 & 6.2 & 6.5 & 6.1 & 6.7 \\
\hline 1 (ns) & 6.6 & 6.9 & 6.4 & 6.2 & 6.3 & 6.3 \\
\hline $7(\mathrm{~ns})$ & 6.3 & 6.8 & 6.4 & 6.5 & 6.2 & 6.2 \\
\hline 15 (ns) & 6.9 & 6.7 & 6.8 & 6.6 & 6.4 & 6.7 \\
\hline 30 (ns) & 6.8 & 6.3 & 6.9 & 6.1 & 6.4 & 6.2 \\
\hline \multicolumn{7}{|c|}{$\mathrm{T}\left({ }^{\circ} \mathrm{C}\right)$} \\
\hline \multicolumn{7}{|c|}{ Crop season $2012 / 13$} \\
\hline Day & T1 (B) & $\mathrm{T} 2(\mathrm{~B})$ & T3 (B) & T4 (B) & T5 (B) & T6 (A) \\
\hline 0 (a) & 35 & 32.8 & 31.3 & 31.4 & 32 & 35.6 \\
\hline $1(b)$ & 27.2 & 31.2 & 28.1 & 28.3 & 28.6 & 35.2 \\
\hline 15 (b) & 26.2 & 29.2 & 29 & 29 & 28.5 & 33 \\
\hline $30(b)$ & 28 & 27.5 & 29.4 & 29.5 & 29.7 & 34.6 \\
\hline \multicolumn{7}{|c|}{ Crop season $2013 / 14$} \\
\hline Day & T1 (B) & T2 (B) & T3 (B) & T4 (B) & T5 (B) & T6 (A) \\
\hline $0(\mathrm{c})$ & 27.2 & 27.7 & 27.1 & 27.2 & 26.6 & 31.6 \\
\hline 1 (b) & 31.2 & 31.1 & 31.2 & 31 & 30.8 & 35.9 \\
\hline 7 (c) & 27.5 & 27.6 & 27.5 & 27.7 & 27.6 & 31.3 \\
\hline $15(b)$ & 31.8 & 32.3 & 32.3 & 32.4 & 32.6 & 34.4 \\
\hline 30 (a) & 34.6 & 34.9 & 34.3 & 34.8 & 33.8 & 38.5 \\
\hline
\end{tabular}

* Means followed by the same letters do not statistically differ among themselves (capital letters in the column and lower case in the line) according to Tukey's test with 5\% of error probability. Ns: non-significant. 
TABLE II

Pesticide concentration in the water $\left(\mu \mathrm{g} \mathrm{L}^{-1}\right)$ and in the soil $\left(\mathrm{mg} \mathrm{kg}^{-1}\right)$. Day 0: 5 min after pesticide applications; day 1: 1 day after pesticide applications; day 7: 7 days after pesticide applications; day 15: 15 days after pesticide applications; day 30: 30 days after pesticide applications. LOQ: limit of quantification of the method; nd: non-detected.

\begin{tabular}{|c|c|c|c|c|c|c|c|c|c|c|c|}
\hline \multicolumn{12}{|c|}{ Crop season $2012 / 13$} \\
\hline & & \multicolumn{5}{|c|}{ Concentration in water $\left(\mu \mathrm{g} \mathrm{L}^{-1}\right)$} & \multicolumn{5}{|c|}{ Concentration in the soil $\left(\mathrm{mg} \mathrm{kg}^{-1}\right)$} \\
\hline & & \multicolumn{5}{|c|}{ Sampling days } & \multicolumn{5}{|c|}{ Sampling days } \\
\hline Treatment & Active Principle & 0 & 1 & 7 & 15 & 30 & 0 & 1 & 7 & 15 & 30 \\
\hline $\mathrm{T} 1$ & Trifloxystrobin & 22.6 & 1.4 & - & n.d & n.d & - & n.d & - & n.d & $\overline{\text { n.d }}$ \\
\hline $\mathrm{T} 1$ & Tebuconazole & 78.4 & 16.4 & - & 4.2 & 3.8 & - & 0.01 & - & n.d & n.d \\
\hline $\mathrm{T} 2$ & Tricyclazole & 192.9 & 36.7 & - & 4.1 & 3.4 & - & n.d & - & n.d & n.d \\
\hline T3 & Lambda-cyhalothrin & 1.6 & n.d & - & n.d & n.d & - & n.d & - & n.d & n.d \\
\hline $\mathrm{T} 3$ & Thiamethoxam & 21.2 & $<$ LOQ & - & n.d & n.d & - & n.d & - & n.d & n.d \\
\hline $\mathrm{T} 4$ & Diflubenzuron & 109.4 & n.d & - & n.d & n.d & - & n.d & - & n.d & n.d \\
\hline \multicolumn{12}{|c|}{ Crop season $2013 / 14$} \\
\hline & & \multicolumn{5}{|c|}{ Concentration in water $\left(\mu \mathrm{g} \mathrm{L}^{-1}\right)$} & \multicolumn{5}{|c|}{ Concentration in the soil $\left(\mathrm{mg} \mathrm{kg}^{-1}\right)$} \\
\hline & & \multicolumn{5}{|c|}{ Sampling days } & \multicolumn{5}{|c|}{ Sampling days } \\
\hline Treatment & Active Principle & 0 & 1 & 7 & 15 & 30 & 0 & 1 & 7 & 15 & 30 \\
\hline $\mathrm{T} 1$ & Trifloxystrobin & $<\mathrm{LOQ}$ & $<$ LOQ & n.d & n.d & n.d & - & n.d & n.d & n.d & n.d \\
\hline $\mathrm{T} 1$ & Tebuconazole & 74.7 & $<$ LOQ & 9.5 & 2.5 & 0.8 & - & n.d & n.d & n.d & n.d \\
\hline $\mathrm{T} 2$ & Tricyclazole & 48.9 & 40.3 & 16 & 6.1 & 6 & - & n.d & n.d & n.d & n.d \\
\hline $\mathrm{T} 3$ & Lambda-cyhalothrin & 261.2 & n.d & n.d & n.d & n.d & - & n.d & n.d & n.d & n.d \\
\hline $\mathrm{T} 3$ & Thiamethoxam & 31.7 & 12 & 6.6 & 1.5 & 0.2 & - & n.d & n.d & n.d & n.d \\
\hline $\mathrm{T} 4$ & Diflubenzuron & 9.1 & 7.8 & 0.6 & n.d & n.d & - & 5.9 & n.d & n.d & n.d \\
\hline
\end{tabular}

of trifloxystrobin and tebuconazole, and also between the pesticides themselves.

Tricyclazole presented the same behavior as tebuconazole; it was detected in the water until 30 days after the applications. However, it was not found in the soil in both sampling years.

Lambda-cyhalothrin was detected in the water only in the first assessment, 5 min after the applications, and it was not found in the soil in both study years. Residues of the active principle thiamethoxam were detected in the water in the first year until 1 day after the applications. However, they were detected until the $30^{\text {th }}$ day after the applications in the second year, and they were not found in the soil in both years. There was a significant positive correlation between lambdacyhalothrin and thiamethoxam in the water in both years (Tables V and VI).

Similarly to lambda-cyhalothrin, diflubenzuron was detected in the water only in the first evaluation at 5 min after the applications, in the first year. In the second year, it was detected until the $15^{\text {th }}$ day after the applications, and it was also found in the soil 1 day after the applications, at a $5.9 \mathrm{mg} \mathrm{kg}^{-1}$ concentration. In the first year, there was a significant positive correlation between diflubenzuron concentration and water $\mathrm{pH}$.

\section{AQUATIC BENTHIC MACROINVERTEBRATES}

Four phyla were identified in the samples: Arthropoda, Annelida, Nematoda and Mollusca. The Arthropoda phylum was composed of the Insecta class, Diptera order (Chironomidae, Ceratopogonidae and Tipulidae families), Odonata (Libellulidae and Coenagrionidae families), Coleoptera (Dryopidae, Noteridae, Curculionidae - larvae and adults, Ditiscidae - larvae and adults, Gyrinidae and Hidrophilidae families), Tricoptera (Hydroptilidae family), Lepidoptera, Hemiptera (Veliidae family); Entognatha class (Collembola order) and of the Crustacea sub-phylum, Ostracoda class. The Annelida phylum was composed of 
two classes: Oligochaeta and Hirudinea, and the Mollusca phylum was composed of the Gastropoda class, Mesogastropoda order (Ampularidae family, Pomacea sp. genus) and Basommatophora (Planorbidae family, Biomphalaria sp. genus) (Table III).

TABLE III

Density (organisms $/ \mathrm{m}^{2}$ ) of benthic macroinvertebrates in each treatment, in both years of study. T1 - trifloxystrobin + tebuconazole; T2 - tricyclazole; T3 - lambda-cyhalothrin + thiamethoxam; T4 - diflubenzuron; T5 - control with rice plants; T6 - control without rice plants. CV: coefficient of variation.

\begin{tabular}{|c|c|c|c|c|c|c|c|c|c|c|c|c|}
\hline \multirow{3}{*}{ Taxa } & \multicolumn{6}{|c|}{ Crop season $2012 / 13$} & \multicolumn{6}{|c|}{ Crop season 2013/14 } \\
\hline & \multicolumn{6}{|c|}{ Treatments } & \multicolumn{6}{|c|}{ Treatments } \\
\hline & $\mathrm{T} 1$ & $\mathrm{~T} 2$ & T3 & $\mathrm{T} 4$ & T5 & T6 & $\mathrm{T} 1$ & $\mathrm{~T} 2$ & T3 & T4 & T5 & T6 \\
\hline \multicolumn{13}{|l|}{ ARTHROPODA } \\
\hline \multicolumn{13}{|l|}{ INSECTA } \\
\hline \multicolumn{13}{|l|}{ Diptera } \\
\hline Chironomidae & 758 & 325.8 & 458 & 461.2 & 567.7 & 1506.4 & 15.3 & 53.8 & 46.1 & 23 & 25.6 & 69.2 \\
\hline Ceratopogonidae & 35.4 & 54.8 & 141.9 & 41.9 & 145.1 & 1193.5 & 23 & 28.2 & 12.8 & 46.1 & 12.8 & 417.9 \\
\hline Tipulidae & 3.2 & 3.2 & 9.6 & 6.4 & 6.4 & 25.8 & 2.5 & 7.6 & 2.5 & 5.1 & 7.6 & 0 \\
\hline \multicolumn{13}{|l|}{ Odonata } \\
\hline Libellulidae & 3.2 & 0 & 3.2 & 3.2 & 0 & 80.6 & 0 & 2.5 & 0 & 0 & 0 & 15.3 \\
\hline Coenagrionidae & 16.1 & 0 & 0 & 0 & 0 & 6.4 & 2.5 & 2.5 & 2.5 & 0 & 2.5 & 17.9 \\
\hline \multicolumn{13}{|l|}{ Coleoptera } \\
\hline Dryopidae & 19.3 & 12.9 & 12.9 & 25.8 & 12.9 & 6.4 & 0 & 0 & 0 & 0 & 0 & 0 \\
\hline Noteridae & 0 & 3.2 & 0 & 6.4 & 3.2 & 0 & 0 & 0 & 0 & 0 & 0 & 0 \\
\hline Curculionidae (larvas) & 6.4 & 3.2 & 6.4 & 0 & 9.6 & 80.6 & 30.7 & 43.5 & 61.5 & 56.4 & 35.9 & 225.6 \\
\hline Curculionidae (adultos) & 0 & 0 & 0 & 0 & 0 & 0 & 0 & 7.6 & 7.6 & 5.1 & 5.1 & 0 \\
\hline Ditiscidae (larvas) & 3.2 & 0 & 0 & 0 & 0 & 0 & 0 & 0 & 0 & 0 & 0 & 0 \\
\hline Ditiscidae (adultos) & 0 & 0 & 0 & 6.4 & 6.4 & 16.1 & 0 & 0 & 0 & 0 & 0 & 0 \\
\hline Gyrinidae & 6.4 & 9.6 & 0 & 3.2 & 6.4 & 32.2 & 0 & 0 & 0 & 0 & 0 & 0 \\
\hline Hidrophilidae & 0 & 0 & 0 & 3.2 & 0 & 19.3 & 2.5 & 0 & 0 & 0 & 0 & 0 \\
\hline \multicolumn{13}{|l|}{ Tricoptera } \\
\hline Hydroptilidae & 0 & 3.2 & 0 & 0 & 0 & 0 & 2.5 & 10.2 & 7.6 & 12.8 & 17.9 & 2.5 \\
\hline Lepidoptera & 0 & 0 & 0 & 0 & 3.2 & 0 & 0 & 0 & 2.5 & 0 & 0 & 0 \\
\hline \multicolumn{13}{|l|}{ Hemiptera } \\
\hline Veliidae & 0 & 6.4 & 3.2 & 0 & 0 & 0 & 0 & 0 & 0 & 5.1 & 0 & 0 \\
\hline Collembola & 0 & 3.2 & 0 & 0 & 0 & 0 & 0 & 2.5 & 0 & 0 & 0 & 0 \\
\hline \multicolumn{13}{|l|}{ CRUSTACEA } \\
\hline OSTRACODA & 0 & 9.6 & 0 & 0 & 0 & 6.4 & 0 & 0 & 0 & 0 & 0 & 0 \\
\hline \multicolumn{13}{|l|}{$\begin{array}{l}\text { ANNELIDA } \\
\end{array}$} \\
\hline OLIGOCHAETA & 45.1 & 80.6 & 138.7 & 83.8 & 119.3 & 135.4 & 94.8 & 87.1 & 51.2 & 100 & 28.2 & 523 \\
\hline HIRUDINEA & 0 & 19.3 & 41.9 & 6.4 & 3.2 & 0 & 0 & 0 & 0 & 0 & 0 & 0 \\
\hline NEMATODA & 67.7 & 206.4 & 164.5 & 351.6 & 251.6 & 145.1 & 12.8 & 2.5 & 7.6 & 5.1 & 0 & 56.4 \\
\hline \multicolumn{13}{|l|}{ MOLLUSCA } \\
\hline \multicolumn{13}{|l|}{ GASTROPODA } \\
\hline \multicolumn{13}{|l|}{ Mesogastropoda } \\
\hline \multicolumn{13}{|l|}{ Ampularidae } \\
\hline Pomacea sp. & 35.4 & 16.1 & 45.1 & 38.7 & 3.2 & 12.9 & 12.8 & 7.6 & 35.8 & 20.5 & 0 & 5.1 \\
\hline \multicolumn{13}{|l|}{ Basommatophora } \\
\hline \multicolumn{13}{|l|}{ Planorbidae } \\
\hline Biomphalaria sp. & 29 & 103.2 & 96.7 & 277.4 & 83.8 & 9.6 & 0 & 0 & 12.8 & 0 & 0 & 0 \\
\hline Total & 1029 & 861.2 & 1122 & 1316 & 1222 & 3277 & 200 & 256.4 & 251.2 & 279.4 & 135.9 & 1333 \\
\hline CV (\%) & 9.3 & 16.6 & 24 & 8.9 & 11.1 & 28.7 & 24.1 & 14.1 & 16.5 & 25.2 & 12.8 & 11 \\
\hline
\end{tabular}


In the first crop season, a total density of 8.827 organisms $/ \mathrm{m}^{2}$ was found for all treatments and sampling days, compared to 2.454 organisms/ $\mathrm{m}^{2}$ found in the second crop season. There was a significant difference for density among the treatments; however, there was no difference for sampling days, in both crop seasons (Table IV).

In both crop seasons, there was higher density of benthic organisms in the control treatment without rice plants (T6), compared to the other treatments, with rice plants. For the first year, density was lower in treatments T1, T2 and T3; however, it was not different from T4 and T5 (control), which presented a similar average density. In the second year, all treatments, except for the control without rice plants (T6), presented similar density among themselves.

There was no significant correlation between the density of the organisms and pesticide concentrations in water, for both crop seasons. In the first year, there was a significant correlation between the organisms in $\mathrm{T} 1$ and oxygen dissolved in water. The contrary was observed for T5 (control), in which the density showed a significant

TABLE IV

Variance analysis of two criteria for the density of benthic macroinvertebrates in both crop seasons. T1 - trifloxystrobin + tebuconazole; T2 - tricyclazole; T3 - lambda-cyhalothrin + thiamethoxam; T4 - diflubenzuron; T5 - control with rice plants; T6 - control without rice plants.

\begin{tabular}{ccc}
\hline \multirow{2}{*}{ Treatments } & \multicolumn{2}{c}{ Density $\left(\right.$ org $\left./ \mathrm{m}^{2}\right)$} \\
\cline { 2 - 3 } & Crop season $2012 / 13$ & Crop season $2013 / 14$ \\
T1 & $1.029 \mathrm{~b}^{*}$ & $200 \mathrm{~b}$ \\
$\mathrm{~T} 2$ & $861 \mathrm{~b}$ & $256 \mathrm{~b}$ \\
$\mathrm{~T} 3$ & $1.122 \mathrm{~b}$ & $251 \mathrm{~b}$ \\
$\mathrm{~T} 4$ & $1.316 \mathrm{ab}$ & $279 \mathrm{~b}$ \\
$\mathrm{~T} 5$ & $1.222 \mathrm{ab}$ & $135 \mathrm{~b}$ \\
T6 & $3.277 \mathrm{a}$ & $1.333 \mathrm{a}$ \\
Total & 8.827 & 2.454 \\
F treatment & 3.68 & 31.66 \\
p treatment & 0.02 & $<.0001$ \\
F sampling days & 1 & 1.62 \\
p sampling days & 0.41 & 0.2073 \\
\hline
\end{tabular}

* Lower case letters compare the treatments among themselves in each crop season. Means followed by the same letters do not statistically differ among themselves according to Tukey's test with 5\% of error probability.

TABLE V

Correlation matrix for density $\left(\mathrm{org} / \mathrm{m}^{2}\right)$ of benthic macroinvertebrates, physical-chemical parameters and pesticide concentration in water, at crop season 2012/13. T1 - trifloxystrobin + tebuconazole; T2 - tricyclazole; T3 - lambdacyhalothrin + thiamethoxam; T4 - diflubenzuron; T5 - control with rice plants; T6 - control without rice plants. DO: dissolved oxygen; pH: potential hydrogen; T: temperature; Tfx: trifloxystrobin; Tbc: tebuconazole; Tcc: tricyclazole; $\lambda$ : lambda-cyhalothrin; Tmx: thiamethoxam; Dfb: diflubenzuron. Numbers in bold represent $\mathbf{p} \leq \mathbf{0 . 0 5}$.

\begin{tabular}{lcccccc}
\hline Crop season $2012 / 13$ & Density T1 & $\mathrm{DO}\left(\mathrm{mg} \mathrm{L}^{-1}\right)$ & $\mathrm{pH}$ & $\mathrm{T}\left({ }^{\circ} \mathrm{C}\right)$ & $\mathrm{Tfx}\left(\mu \mathrm{g} \mathrm{L}^{-1}\right)$ & $\mathrm{Tbc}\left(\mu \mathrm{g} \mathrm{L}^{-1}\right)$ \\
\hline Density T1 & 1 & --- & --- & --- & --- & --- \\
$\mathrm{DO}\left(\mathrm{mg} \mathrm{L}^{-1}\right)$ & $\mathbf{0 . 9 8}$ & 1 & --- & -- & -- & --- \\
$\mathrm{pH}$ & -0.06 & 0.08 & 1 & --- & --- & --- \\
$\mathrm{T}\left({ }^{\circ} \mathrm{C}\right)$ & 0.11 & 0.25 & 0.77 & 1 & --- & --- \\
$\mathrm{Tfx}\left(\mu \mathrm{g} \mathrm{L}^{-1}\right)$ & -0.03 & 0.11 & 0.85 & $\mathbf{0 . 9 8}$ & 1 & --- \\
$\operatorname{Tbc}\left(\mu \mathrm{g} \mathrm{L}^{-1}\right)$ & -0.12 & 0.02 & 0.82 & $\mathbf{0 . 9 7}$ & $\mathbf{0 . 9 9}$ & 1 \\
\hline
\end{tabular}


TABLE V (continuation)

\begin{tabular}{|c|c|c|c|c|c|c|}
\hline & Density T2 & $\mathrm{DO}\left(\mathrm{mg} \mathrm{L}^{-1}\right)$ & $\mathrm{pH}$ & $\mathrm{T}\left({ }^{\circ} \mathrm{C}\right)$ & $\operatorname{Tcc}\left(\mu \mathrm{g} \mathrm{L}^{-1}\right)$ & \\
\hline Density T2 & 1 & --- & --- & --- & --- & \\
\hline $\mathrm{DO}\left(\mathrm{mg} \mathrm{L}^{-1}\right)$ & -0.31 & 1 & --- & --- & --- & \\
\hline $\mathrm{pH}$ & -0.77 & 0.60 & 1 & --- & --- & \\
\hline $\mathrm{T}\left({ }^{\circ} \mathrm{C}\right)$ & -0.10 & -0.36 & 0.38 & 1 & --- & \\
\hline \multirow[t]{2}{*}{$\operatorname{Tcc}\left(\mu \mathrm{g} \mathrm{L}^{-1}\right)$} & 0.01 & 0.10 & 0.53 & 0.84 & 1 & \\
\hline & Density T3 & $\mathrm{DO}\left(\mathrm{mg} \mathrm{L}^{-1}\right)$ & $\mathrm{pH}$ & $\mathrm{T}\left({ }^{\circ} \mathrm{C}\right)$ & $\lambda\left(\mu \mathrm{g} \mathrm{L}^{-1}\right)$ & $\operatorname{Tmx}\left(\mu \mathrm{g} \mathrm{L}^{-1}\right)$ \\
\hline Density T3 & 1 & --- & --- & --- & --- & --- \\
\hline $\mathrm{DO}\left(\mathrm{mg} \mathrm{L}^{-1}\right)$ & -0.82 & 1 & --- & --- & --- & --- \\
\hline $\mathrm{pH}$ & 0.74 & -0.98 & 1 & --- & --- & --- \\
\hline $\mathrm{T}\left({ }^{\circ} \mathrm{C}\right)$ & 0.25 & -0.64 & 0.60 & 1 & --- & --- \\
\hline$\lambda\left(\mu \mathrm{g} \mathrm{L}^{-1}\right)$ & -0.11 & -0.45 & 0.57 & 0.55 & 1 & --- \\
\hline \multirow[t]{2}{*}{$\operatorname{Tmx}\left(\mu \mathrm{g} \mathrm{L}^{-1}\right)$} & -0.11 & -0.45 & 0.57 & 0.55 & 1 & 1 \\
\hline & Density T4 & $\mathrm{DO}\left(\mathrm{mg} \mathrm{L}^{-1}\right)$ & $\mathrm{pH}$ & $\mathrm{T}\left({ }^{\circ} \mathrm{C}\right)$ & $\mathrm{Dfb}\left(\mu \mathrm{g} \mathrm{L}^{-1}\right)$ & \\
\hline Density T4 & 1 & --- & --- & --- & --- & \\
\hline $\mathrm{DO}\left(\mathrm{mg} \mathrm{L}^{-1}\right)$ & -0.06 & 1 & --- & --- & --- & \\
\hline $\mathrm{pH}$ & -0.58 & -0.50 & 1 & --- & --- & \\
\hline $\mathrm{T}\left({ }^{\circ} \mathrm{C}\right)$ & -0.47 & -0.72 & 0.41 & 1 & --- & \\
\hline \multirow[t]{2}{*}{$\mathrm{Dfb}\left(\mu \mathrm{g} \mathrm{L}^{-1}\right)$} & -0.77 & -0.44 & 0.96 & 0.55 & 1 & \\
\hline & Density T5 & $\mathrm{DO}\left(\mathrm{mg} \mathrm{L}^{-1}\right)$ & $\mathrm{pH}$ & $\mathrm{T}\left({ }^{\circ} \mathrm{C}\right)$ & & \\
\hline Density T5 & 1 & --- & --- & --- & & \\
\hline $\mathrm{DO}\left(\mathrm{mg} \mathrm{L}^{-1}\right)$ & -0.99 & 1 & --- & --- & & \\
\hline $\mathrm{pH}$ & 0.32 & -0.30 & 1 & --- & & \\
\hline \multirow[t]{2}{*}{$\mathrm{T}\left({ }^{\circ} \mathrm{C}\right)$} & 0.89 & -0.84 & 0.55 & 1 & & \\
\hline & Density T6 & $\mathrm{DO}\left(\mathrm{mg} \mathrm{L}^{-1}\right)$ & $\mathrm{pH}$ & $\mathrm{T}\left({ }^{\circ} \mathrm{C}\right)$ & & \\
\hline Density T6 & 1 & --- & --- & --- & & \\
\hline $\mathrm{DO}\left(\mathrm{mg} \mathrm{L}^{-1}\right)$ & -0.60 & 1 & --- & --- & & \\
\hline $\mathrm{pH}$ & 0.71 & -0.57 & 1 & --- & & \\
\hline $\mathrm{T}\left({ }^{\circ} \mathrm{C}\right)$ & 0.51 & -0.99 & 0.54 & 1 & & \\
\hline
\end{tabular}

TABLE VI

Correlation matrix for density $\left(\mathrm{org} / \mathrm{m}^{2}\right)$ of benthic macroinvertebrates, physical-chemical parameters and pesticide concentration in water, at crop season 2013/14. T1 - trifloxystrobin + tebuconazole; T2 - tricyclazole; T3 - lambdacyhalothrin + thiamethoxam; T4 - diflubenzuron; T5 - control with rice plants; T6 - control without rice plants. DO: dissolved oxygen; pH: potential hydrogen; T: temperature; Tbc: tebuconazole; Tcc: tricyclazole; $\lambda$ : lambda-cyhalothrin; Tmx: thiamethoxam; Dfb: diflubenzuron. Numbers in bold represent $\mathbf{p} \leq \mathbf{0 . 0 5}$.

\begin{tabular}{lccccc}
\hline Crop season $2013 / 14$ & Density T1 & $\mathrm{OD}\left(\mathrm{mg} \mathrm{L}^{-1}\right)$ & $\mathrm{pH}$ & $\mathrm{T}\left({ }^{\circ} \mathrm{C}\right)$ & $\mathrm{Tbc}\left(\mu \mathrm{g} \mathrm{L}^{-1}\right)$ \\
\hline Density T1 & 1 & --- & --- & --- & --- \\
$\mathrm{DO}\left(\mathrm{mg} \mathrm{L}^{-1}\right)$ & -0.01 & 1 & --- & --- & --- \\
$\mathrm{pH}$ & 0.47 & -0.72 & 1 & --- & --- \\
$\mathrm{T}\left({ }^{\circ} \mathrm{C}\right)$ & 0.06 & $-\mathbf{0 . 8 9}$ & $\mathbf{0 . 8 7}$ & 1 & --- \\
$\mathrm{Tbc}\left(\mu \mathrm{g} \mathrm{L}^{-1}\right)$ & 0.43 & 0.46 & -0.51 & -0.66 & 1 \\
\hline & Density T2 & $\mathrm{DO}\left(\mathrm{mg} \mathrm{L}^{-1}\right)$ & $\mathrm{pH}$ & $\mathrm{T}\left({ }^{\circ} \mathrm{C}\right)$ & $\mathrm{Tcc}\left(\mu \mathrm{g} \mathrm{L}^{-1}\right)$ \\
\hline Density T2 & 1 & --- & --- & --- & --- \\
DO $\left(\mathrm{mg} \mathrm{L}^{-1}\right)$ & -0.43 & 1 & -- & --- & --- \\
$\mathrm{pH}$ & -0.19 & 0.73 & 1 & --- & --- \\
$\mathrm{T}\left({ }^{\circ} \mathrm{C}\right)$ & 0.45 & $-\mathbf{0 . 9 7}$ & -0.65 & 1 & --- \\
Tcc $\left(\mu \mathrm{g} \mathrm{L}^{-1}\right)$ & 0.24 & 0.48 & 0.52 & -0.59 & 1 \\
\hline
\end{tabular}


TABLE VI (continuation)

\begin{tabular}{|c|c|c|c|c|c|c|}
\hline & Density T3 & $\mathrm{DO}\left(\mathrm{mg} \mathrm{L}^{-1}\right)$ & $\mathrm{pH}$ & $\mathrm{T}\left({ }^{\circ} \mathrm{C}\right)$ & $\lambda\left(\mu \mathrm{g} \mathrm{L}^{-1}\right)$ & $\operatorname{Tmx}\left(\mu \mathrm{g} \mathrm{L}^{-1}\right)$ \\
\hline Density T3 & 1 & --- & --- & --- & --- & --- \\
\hline $\mathrm{DO}\left(\mathrm{mg} \mathrm{L}^{-1}\right)$ & -0.33 & 1 & --- & --- & --- & --- \\
\hline $\mathrm{pH}$ & 0.45 & -0.76 & 1 & --- & --- & --- \\
\hline $\mathrm{T}\left({ }^{\circ} \mathrm{C}\right)$ & 0.39 & -0.96 & 0.89 & 1 & --- & --- \\
\hline$\lambda\left(\mu \mathrm{g} \mathrm{L}^{-1}\right)$ & 0.10 & 0.46 & -0.64 & -0.60 & 1 & --- \\
\hline \multirow[t]{2}{*}{$\operatorname{Tmx}\left(\mu \mathrm{g} \mathrm{L}^{-1}\right)$} & -0.14 & 0.54 & -0.85 & -0.72 & 0.93 & 1 \\
\hline & Density T4 & $\mathrm{DO}\left(\mathrm{mg} \mathrm{L}^{-1}\right)$ & $\mathrm{pH}$ & $\mathrm{T}\left({ }^{\circ} \mathrm{C}\right)$ & $\mathrm{Dfb}\left(\mu \mathrm{g} \mathrm{L}^{-1}\right)$ & \\
\hline Density T4 & 1 & --- & --- & --- & --- & \\
\hline $\mathrm{DO}\left(\mathrm{mg} \mathrm{L}^{-1}\right)$ & 0.25 & 1 & --- & --- & --- & \\
\hline pH & 0.21 & 0.64 & 1 & --- & --- & \\
\hline $\mathrm{T}\left({ }^{\circ} \mathrm{C}\right)$ & -0.46 & -0.94 & -0.57 & 1 & --- & \\
\hline \multirow[t]{2}{*}{$\operatorname{Dfb}\left(\mu \mathrm{g} \mathrm{L}^{-1}\right)$} & 0.63 & 0.15 & -0.06 & -0.47 & 1 & \\
\hline & Density T5 & $\mathrm{DO}\left(\mathrm{mg} \mathrm{L}^{-1}\right)$ & $\mathrm{pH}$ & $\mathrm{T}\left({ }^{\circ} \mathrm{C}\right)$ & & \\
\hline Density T5 & 1 & --- & --- & --- & & \\
\hline $\mathrm{DO}\left(\mathrm{mg} \mathrm{L}^{-1}\right)$ & 0.37 & 1 & --- & --- & & \\
\hline $\mathrm{pH}$ & -0.33 & -0.69 & 1 & --- & & \\
\hline \multirow[t]{2}{*}{$\mathrm{T}\left({ }^{\circ} \mathrm{C}\right)$} & -0.28 & -0.80 & 0.97 & 1 & & \\
\hline & Density T6 & $\mathrm{DO}\left(\mathrm{mg} \mathrm{L}^{-1}\right)$ & $\mathrm{pH}$ & $\mathrm{T}\left({ }^{\circ} \mathrm{C}\right)$ & & \\
\hline Density T6 & 1 & --- & --- & --- & & \\
\hline $\mathrm{DO}\left(\mathrm{mg} \mathrm{L}^{-1}\right)$ & 0.81 & 1 & --- & --- & & \\
\hline $\mathrm{pH}$ & -0.22 & 0.07 & 1 & --- & & \\
\hline $\mathrm{T}\left({ }^{\circ} \mathrm{C}\right)$ & -0.48 & -0.78 & -0.37 & 1 & & \\
\hline
\end{tabular}

negative correlation with oxygen, which may have occurred as a result of the rise in water temperature, favoring the increase in density and causing a reduction in the dissolved oxygen. Such reduction, however, was not drastic, with adequate levels to the development of the organisms.

\section{DISCUSSION}

Pesticide application did not change the physicalchemical properties of water in the experimental plots, since all assessed parameters were maintained at adequate levels for the development of the organisms. There was a significant negative correlation between dissolved oxygen in water and temperature due to the fact that the solubility of gases in water decreases with increased temperature (Fiorucci and Benedetti Filho 2005).

Previous studies in the same experimental area resulted in smaller rates of dissolved oxygen, whereas Golombieski et al. (2008) found values of $0.6-2.2 \mathrm{mg} \mathrm{L}^{-1}$ and Reimche et al. (2008) found values of $2.4-4.6 \mathrm{mg} \mathrm{L}^{-1}$, which indicated good conditions of oxygen in water in the current studied years. The $\mathrm{pH}$ values of water were maintained adequate for this type of environment, according to Resolution $357 / 2005$ by CONAMA (National Environment Council), which must not be below 6.0 (Brasil 2005).

Although there were not any significant effects of the pesticides over the density of benthic organisms, considering the dosages in use and frequency, tebuconazole, tricyclazole and thiamethoxam presented water persistence up to 30 days after their application. Photodegrading is an unlikely degradation route of tebuconazole in the environment, because it occurs very slowly due to the fact that absorption is very low at wavelengths above $290 \mathrm{~nm}$ (FAO 1994). Recent studies have identified approximately 22 known transformation 
products and 12 still unknown transformation products of degradation of tebuconazole in the soil (Storck et al. 2015). Similarly, enantioselectivity is a process that has been recently studied, and it may contribute to toxicity in the natural environment. The enantioselectivity of tebuconazole was assessed over Scenedesmus obliquus, Daphnia magna and Danio rerio, whereas R - (-) tebuconazole was 1.4 to 5.9 times more toxic than $\mathrm{S}-(+)$ - tebuconazole ( $\mathrm{Li}$ et al. 2015). This process, however, seems to be related to soil properties ( $\mathrm{Li}$ et al. 2015) and so far there was no significant enantioselective degradation for tebuconazole in sterilized conditions (Zhang et al. 2015), showing the importance of organic matter in the enantioselective degradation of the fungicide.

Tricyclazole is considered to be a persistent fungicide in the environment, with high risk of environmental contamination (Padovani et al. 2006). The dissipation of penoxsulam, tricyclazole and profoxydim in rice fields was verified in laboratory and field conditions, whereas tricyclazole was the most resistant pesticide with DT50 of 44.5 - 84.6 days in the field and 197 days in the laboratory (Tsochatzis et al. 2013). GarcíaJaramillo et al. (2014) observed that organic matter plays an important role in the adsorption of tricyclazole to the soil particles. There was maximum adsorption and minimum desorption of tricyclazole in soils with high content of clay and organic carbon, showing that the phreatic zone contamination may be minimized when tricyclazole is applied in rice cultivation areas whose soils have these characteristics (Kumar et al. 2015).

The thiamethoxam active principle belongs to the neonicotinoid class, which is considered to be persistent in the environment, with high leaching capacity and high toxicity for several invertebrate species (Morrissey et al. 2015). In this study, thiamethoxam presented elevated water persistence. It was detected until 30 days after application, whereas soil residues were not detected. These results corroborate with those found by Scorza Jr and Rigitano (2012), who stated that thiamethoxam presents high solubility in water and low sorption to the soil. Results obtained in the same experimental area show the presence of thiamethoxam in the irrigation water of rice up to 40 days after application (Teló et al. 2015).

More than $75 \%$ of the total density of the benthic organisms collected during both sampling years, was found within the first year of the study. Little soil disturbance in the rice paddy crop prior to the first year may have favored the establishment of species in this period, while in the second year of the experiment, the construction of bunds, destruction of bunds and soil leveling for fallow purposes and the construction of new bunds in the second year of the experiment may have contributed to a decrease in floodplains for the establishment of organisms. In addition, the climate may have favored the colonization in the first year, because in this period, the average water temperature was lower when compared to the second year $\left(1^{\circ} \mathrm{C}\right.$ approximately) and there was a higher rate of dissolved oxygen in water $(7.3 \mathrm{mg}$ $\mathrm{L}^{-1}$ overall mean for the first year and $6.9 \mathrm{mg} \mathrm{L}^{-1}$ in the second year). According to Willming et al. (2013), fluctuations in temperature may influence the ability of the organisms to detoxify xenobiotics, thus changing the absorption of contaminants, the rates of elimination and biotransformation, resulting in changes in the toxicity and in the toxicodynamic processes of pesticides in the organisms. Camargo (2010) cites that daily oscillations in temperature may not pose stress to benthic organisms in larger and more stable habitats than in rice fields. However, for this ecosystem, such changes may be sufficiently drastic to hinder the establishment of $\mathrm{K}$-strategists and, thus, prevent the advance in the successional process.

The richness of the macroinvertebrate fauna found in this research was similar to the one found by other authors in Brazilian rice paddy fields 
(Molozzi et al. 2007, Baumart and Santos 2011), in which dipteran insects of the Chironomidae family were dominant compared to other taxa. A significant abundance of insects from the Chironomidae and Ceratopogonidae families is associated with the fact that such organisms are considered resistant to the environment conditions of oxygen depletion and disturbances associated with rice crop management (Molozzi et al. 2007). Extremely tolerant organisms, represented by Chironomidae larvae, by other Diptera and by the entire Oligochaeta class, are capable of living under anoxic conditions for several hours. Moreover, they are detritivores, i.e. they feed from organic matter deposited in the sediment, which favor their adaptation to several environments. Suhling et al. (2000) highlight that high densities of Chironomidae, Oligochaeta and Mollusca are common in rice paddy fields and the low density of Ephemeroptera, Plecoptera and Trichoptera (EPT) is due to the application of insecticides and high concentration of nitrogen and phosphorus in the soil.

The highest total density of organisms in the control treatment without rice plants (T6), compared to the other treatments, was due to the elevated density of dipteran from the Chironomidae and Ceratopogonidae families, odonates and Curculionidae larvae in this treatment. In this treatment, given the absence of rice plants, there was a higher incidence of aquatic macrophytes, which created an adequate environment for those macroinvertebrates. The presence of aquatic macrophytes favors the high density of Chironomidae in lentic environments (Dornfeld and Fonseca-Gessner 2005, Rosin and Takeda 2007), and, for odonates, it may favor an adequate habitat for the nymphs. Importantly, caddisflies of the Hydroptilidae family were present in the second year, with higher density in the control treatment with rice plants, which are considered sensible to environmental pollution (Molozzi et al. 2007).
Under the conditions in the study, the pesticides did not significantly reduce the density of the organisms compared to the control treatments. This may have occurred due to the fact that only one application of the fungicide or insecticide occurred in each plot in both experimental years, whereas some of the tested pesticides presented low persistence in the water and soil, and those with higher persistence (tebuconazole, tricyclazole, and thiamethoxam) may not have reached a concentration high enough to cause negative impact over the individuals or they quickly degraded because of environmental conditions. Similar to this study, Rossaro et al. (2014) did not find significant negative effects of the tricyclazole fungicide over the aquatic macroinvertebrate community. However, the authors observed low diversity, which was attributed to the short period of rice submersion, as well as to the low content of oxygen and high water temperature during the experiment. Mesléard et al. (2005) compared the macroinvertebrate communities in rice fields of conventional and organic cultivation in France, and they did not find statistic differences in richness, but rather in the abundance of the species, which was correlated with the use of the insecticide fipronil, previously used for the control of Chironomidae larvae.

The present study was aimed at comparing, in experimental plots, a rice management approach with and without pesticides, and without the presence of rice plants, thus simulating a natural environment. The results were the same, although to a lesser extent, as those found by Dalzochio et al. (2016) in a recent study in natural lakes and rice fields with organic and conventional cultivation. The authors registered higher richness and abundance of macroinvertebrates in the natural lakes compared to the rice fields; however, the fields with organic and conventional rice cultivation showed similar taxonomic richness and abundance. They also observed that many taxa only occurred in 
lakes and organic crops, which demonstrates that the organic management of rice fields favors the establishment of some taxa that are only found in natural environments.

In this study, the establishment of a benthic macroinvertebrate community is favored when it is in a natural environment, free of pesticides and without rice plants. However, the application of a single dose of the fungicides trifloxystrobin + tebuconazole and tricyclazole; and of the insecticides lambda-cyhalothrin + thiamethoxam and diflubenzuron, does not cause significant effects over the richness and density of the macroinvertebrates. Tebuconazole, tricyclazole and thiamethoxam presented high persistence in the water of rice paddy fields, and the doses and number of applications of these products in the field should be carefully controlled in order to avoid environmental contamination.

\section{ACKNOWLEDGMENTS}

The authors would like to thank the Coordenação de Aperfeiçoamento de Pessoal de Nível Superior (CAPES) by $\mathrm{PhD}$ scholarship to the first author and to Conselho Nacional de Desenvolvimento Científico e Tecnológico (CNPq) for the financial support through the Universal Project No. 14/2012.

\section{REFERENCES}

AYRES M, AYRES JR M, AYRES DL AND SANTOS AS. 2007. BioEstat 5.0. Aplicações estatísticas nas áreas das ciências biológicas e médicas. Sociedade Civil Mamirauá (MCT). Imprensa Oficial do Estado do Pará.

BAMBARADENIYA CNB, EDIRISINGHE JP, SILVA DN, GUNATILLEKE CVS, RANAWANA KB AND WIJEKOON S. 2004. Biodiversity associated with an irrigated rice agro-ecosystem in Sri Lanka. Biodivers Conserv 13: 1715-1753.

BAUMART JS AND SANTOS S. 2011. The impact of herbicides on benthic organisms in flooded rice fields in Southern Brazil. In: Herbicides and Environmental. Edited by Andreas Kortekamp. Intech, p. 369-382.

BRASIL. 2005. Ministério do Meio Ambiente. Conselho Nacional do Meio Ambiente. Resolução no 357 de 17 de março de 2005. Diário Ofícial da União, 18 março de 2005. Available from: http://www.mma.gov.br/port/conama/res/ res05/res35705.pdf.

CALDAS SS, GONÇALVES FF, PRIMEL EG, PRESTES OD, MARTINS ML AND ZANELLA R. 2011. Principais técnicas de preparo de amostra para a determinação de resíduo de agrotóxicos em água por cromatografia liquida com detecção por arranjo de diodos e por espectrometria de massas. Quím Nova 34: 1604-1617.

CAMARGO BV. 2010. Macroinvertebrados da lavoura de arroz irrigado tratada com agrotóxicos Carbofuran e Penoxulam. Dissertação de Mestrado (Unpublished): Programa de Pós-Graduação em Biodiversidade Animal, Universidade Federal de Santa Maria, RS, Brasil, 47 p.

COSTA C, IDE S AND SIMONKA CC. 2006. Insetos imaturos: metamorfose e identificação. Ribeirão Preto: Holos Editora, $249 \mathrm{p}$.

COUNCE PA, KEISLING TC AND MITCHELL AJ. 2000. A uniform, objective, and adaptive system for expressing rice development. Crop Sci 40: 436-443.

DALZOCHIO MS, BALDIN R, STENERT C AND MALTCHIK L. 2016. Can organic and conventional agricultural systems affect wetland macroivertebrate taxa in rice fields? Basic Appl Ecol 17: 220-229.

DONATO FF, MARTINS ML, MUNARETTO JS, PRESTES OD, ADAIME MB AND ZANELLA R. 2015. Development of a multiresidue method for pesticide analysis in drinking water by solid phase extraction and determination by gas and liquid chromatography with triple quadrupole tandem mass spectrometry. J Braz Chem Soc 26: 2077 2087.

DORNFELD CB AND FONSECA-GESSNER AA. 2005. Fauna de Chironomidae (Diptera) associada à Salvinia sp. e Myriophyllum sp. num reservatório do Córrego do Espraiado, São Carlos, São Paulo, Brasil. Entomol Vect 12: 181-192.

FAO - FOOD AND AGRICULTURE ORGANIZATION OF THE UNITED NATIONS. 1994. Pesticides residues in food: Tebuconazole (188). Available from: http://www. fao.org/fileadmin/templates/agphome/documents/Pests Pesticides/JMPR/Evaluation94/tebucona.pdf.

FERNÁNDEZ HR AND DOMÍNGUEZ E. 2001. Guia para La determinación de losartrópodos bentônicos sudamericanos. Ed. Imprenta Central de la UNT- Tucumán, Argentina, $289 \mathrm{p}$.

FIORUCCI AR AND BENEDETTI FILHO E. 2005. A importância do oxigênio dissolvido em ecossistemas aquáticos. QNEsc 22: 10-16.

GAMBOA M, REYES R AND ARRIVILLAGA J. 2008. Macroinvertebradosbentónicos como bioindicadores de salud ambiental. B Malariol Salud Amb 48: 109-120.

GARCÍA-JARAMILLO M, COX L, CORNEJO J AND HERMOSÍN MC. 2014. Effect of soil organic amendments 
on the behavior of bentazone and tricyclazole. Sci Total Environ 466-467: 906-913.

GOLOMBIESKI JI, MARCHESAN E, BAUMART JS, REIMCHE GB, RESGALLA JR C, STORCK L AND SANTOS S. 2008. Cladocers, Copepods and Rotifers in rice-fish culture handled with metsulfuron-methyl and azimsulfuron herbicides and carbofuran insecticide. Cienc Rural 38: 2097-2102.

GONÇALVES FF, MATOS FS AND ZANELLA R. 2013. Determinação de resíduos de herbicidas em águas de lavoura de arroz irrigado empregando extração em fase sólida e cromatografia líquida de alta efíciência com detecção por arranjo de diodos. Scient Chromatogr 5: 89-100.

GOULART M AND CALLISTO M. 2003. Bioindicadores de qualidade de água como ferramenta em estudos de impacto ambiental. Revista da FAPAM, ano 2, n.1.

KUHLMANN ML, JOHNSCHER-FORNASARO G, OGURA LL AND IMBIMBO HRB. 2012. Protocolo para o biomonitoramento com as comunidades bentônicas de rios e reservatórios do estado de São Paulo. São Paulo: CETESB, $113 \mathrm{p}$.

KUMAR N, MUKHERJEE I AND VARGHESE E. 2015. Adsorption-desorption of tricyclazole: effect of soil types and organic matter. Environ Monit Assess 187: 1-10.

LI Y, DONG F, LIU X, XU J, HAN Y AND ZHENG Y. 2015. Enantioselectivity in tebuconazole and myclobutanil nontarget toxicity and degradation in soils. Chemosphere 122: 145-153.

LIESS M, SCHÄFER RB AND SCHRIEVER CA. 2008. The footprint of pesticide stress in communities-species traits reveal community effects of toxicants. Sci Total Environ 406: 484-490.

MALTCHIK L, ROLON AS, STENERT C, MACHADO IF AND ROCHA O. 2011. Can rice field channels contribute to biodiversity conservation in Southern Brazilian wetlands? Rev Biol Trop 59: 1895-1914.

MARTINS GL, FRIGGI CA, PRESTES OD, VICARI MC, FRIGGI DA, ADAIME MB AND ZANELLA R. 2014. Simultaneous LC-MS/MS determination of imidazolinone herbicides together with other multiclass pesticide residues in soil. Clean - Soil, Air, Water 42: 1441-1449.

MESLÉARD F, GARNERO S, BECK N AND ROSECCHI E. 2005. Uselessness and indirect negative effects of an insecticide on rice field invertebrates. C R Biol 328: 955962.

MOLOZZI J, HEPP LU AND DIAS AS. 2007. Influence of rice crop on the benthic community in Itajaí Valley (Santa Catarina, Brazil). Acta Limnol Bras 19: 383-392.

MORRISSEY CA, MINEAUC P, DEVRIESD JH, SANCHEZ-BAYOE F, LIESSF M, CAVALLAROB MC AND LIBERB K. 2015. Neonicotinoid contamination of global surface waters and associated risk to aquatic invertebrates: A review. Environ Int 74: 291-303.
MUGNAI R, NESSIMIAN JL AND BAPTISTA DF. 2010. Manual de identificação de macroinvertebrados aquáticos do estado do Rio de Janeiro. Rio de Janeiro: Technical Books, $176 \mathrm{p}$.

PADOVANI L, CAPRI E, PADOVANI C, PUGLISI E AND TREVISAN M. 2006. Monitoring tricyclazole residues in rice paddy watersheds. Chemosphere 62: 303-314.

PRESTES OD, FRIGGI CA, ADAIME MB AND ZANELLA R. 2009. QuEChERS - um método moderno de preparo de amostra para determinação multirresíduo de pesticidas em alimentos por métodos cromatográficos acoplados à espectrometria de massas. Quím Nova 32: 1620-1634.

REIMCHE GB, MACHADO SLO, GOLOMBIESKI JI, BAUMART JS, BRAUN N, MARCHESAN E AND ZANELLA R. 2008. Persistência na água e influência de herbicidas utilizados na lavoura arrozeira sobre a comunidade zooplanctônica de Cladocera, Copepoda e Rotifera. Cienc Rural 38: 7-13.

ROSIN GC AND TAKEDA AM. 2007. Larvas de Chironomidae (Diptera) da planície de inundação do Alto Rio Paraná: distribuição e composição em diferentes ambientes e períodos hidrológicos. Acta Sci Biol Sci 29: 57-63.

ROSSARO B, MARZIALI L AND CORTESI P. 2014. The Effects of tricyclazole treatment on aquatic invertebrates in a rice paddy field. Clean - Soil, Air, Water 42: 29-35.

SCORZA JR RP AND RIGITANO RLO. 2012. Sorção, degradação e lixiviação do inseticida tiametoxam em dois solos do Mato Grosso do Sul. Rev Bras Eng Agríc 16: 564-572.

SOSBAI - SOCIEDADE SUL-BRASILEIRA DE ARROZ IRRIGADO. 2012. Arroz irrigado: Recomendações técnicas da pesquisa para o Sul do Brasil. In: Reunião Técnica da Cultura do Arroz Irrigado, 29, Gravatal, 176 p.

STENERT C, MALTCHIK L AND ROCHA O. 2012. Diversity of aquatic invertebrates in rice fields in southern Brazil. Neotrop Biol Conserv 7: 67-77.

STORCK V ET AL. 2015. Identification and characterization of tebuconazole transformation products in soil by combining suspect screening and molecular typology. Environ Pollut 208: 537-545.

SUHLING F, BEFELD S, HÄUSLER M, KATZUR K, LEPKOJUS S AND MESLÉARD F. 2000. Effects of insecticide applications on macroinvertebrate density and biomass in rice-fields in the Rhône-delta, France. Hydrobiologia 431: 69-79.

TELÓ GM, MARCHESAN E, ZANELLA R, OLIVEIRA ML, COELHO LL AND MARTINS ML. 2015. Residues of fungicides and insecticides in rice field. Agron J 107: 851863.

TSOCHATZIS ED, TZIMOU-TSITOURIDOU R, MENKISSOGLU-SPIROUDI U, KARPOUZAS DG AND KATSANTONIS D. 2013. Laboratory and field 
dissipation of penoxsulam, tricyclazole and profoxydim in rice paddy systems. Chemosphere 91: 1049-1057.

WANDSCHEER ACD, BAUMART JS, MARCHESAN E, SANTOS S, AVILA LA, SOARES CF AND PIRES MM. 2016. Suficiência amostral para estudos de impacto ambiental sobre a comunidade de macroinvertebrados bentônicos em arrozais irrigados. Cienc Rural 46: 26-29.
WILLMING MM, QIN G AND MAUL JD. 2013. Effects of environmentally realistic daily temperature variation on pesticide toxicity to aquatic invertebrates. Environ Toxicol Chem 32: 2738-2745.

ZHANG Q, ZHOU L, YANG Y, HUA X, SHI H AND WANG M. 2015. Study on the stereoselective degradation of three triazole fungicides in sediment. Ecotoxicol Environ Saf 117: 1-6. 
